\title{
Pediatric Obstructive Sleep Apnea: A Review of Approach to Management
}

\author{
Sweta Kumari ${ }^{1}$, Jyoti Bagla ${ }^{2}$, Aarushi Singla ${ }^{3}$
}

\begin{abstract}
Pediatric sleep apnea is an underestimated and often ignored childhood morbidity which is on rise with an increasing prevalence of obesity. Obstructive sleep apnea (OSA) constitutes the bulk of the pediatric sleep-disordered breathing with a fewer proportion of children suffering from central or mixed sleep apnea. OSA often leads to growth failure, cardiovascular dysfunction, behavioral problems, poor learning, and quality of life. Systematic evaluation of the apnea along with appropriate management modality gives an opportunity to gain the short-term and long-term health. This review is focused on the management of OSA in the pediatric age group. Treatment decisions are guided by detailed evaluation findings like anatomic malformation, degree of functional impairment, and sleep study findings.
\end{abstract}

Keywords: Adenotonsillectomy, Apnea-hypopnea index, Continuous positive airway pressure, Literature review, Obstructive sleep apnea. Indian Journal of Sleep Medicine (2019): 10.5005/jp-journals-10069-0035

\section{INTRODUCTION}

Sleep is considered as a fundamental necessity for a normal development in childhood. Breathing abnormalities while sleep has the potential to disturb sleep along with its effect on gas exchange. International classification of sleep disorders has delineated the sleep-related breathing disorders (SRBDs) into subsections of central sleep apnea, OSA, sleep-related hypoventilation disorders, and sleep-related hypoxemia disorder. ${ }^{1}$ OSA in children is defined as a disorder of breathing during sleep causing partial or complete upper airway obstruction during sleep, which leads to impaired ventilation and sleep disruption. ${ }^{2}$ Prevalence studies across the globe has shown the prevalence of sleep apnea to range from $1.2 \%$ to $5.7 \%$ depending on the age group and definition used. ${ }^{3-5}$

A suspected case of sleep apnea needs detailed but stepwise evaluation by an otolaryngologist and a sleep medicine specialist (Table 1). It is important to emphasize that pediatric OSA is different from the clinical presentation in adults as it is characterized by more disturbed nocturnal sleep than excessive daytime sleepiness, and more behavioral problems, particularly school problems, hyperactivity, nocturnal enuresis, sleep terrors, depression, insomnia, and psychiatric problems. ${ }^{6}$ Hence, details of case history regarding sleep issues and its effect on breathing, learning, behavior, and school performance should be enquired from parents. Physical examination of the upper airway using scoring systems like the Mallampati score ${ }^{7}$ or the Friedman tonsil scale ${ }^{8}$ is useful in estimating the contribution of the anatomy to the degree of obstructive. ${ }^{9}$ Assessment of coexisting morbidities like obesity, allergic rhinitis, bronchial asthma, and neuromuscular diseases should be done. Recent evidence supporting the clear association and significant overlap in symptomatology and pathophysiology for pediatric OSA and asthma make it important to recognize the overlap and evaluate for the other condition when one is present. ${ }^{10}$ A syndromic evaluation must be done as per clinical findings as it influences prognosis and further investigation. Growth monitoring should always be a part of systemic evaluation as OSA has been seen to have a negative effect on longitudinal growth. ${ }^{11}$ Investigations are done to characterize the apnea episodes and to manage the comorbid conditions like upper airway anatomic anomalies and

\footnotetext{
${ }^{1-3}$ Department of Pediatrics, ESI Hospital and PGIMSR, Basaidarapur, New Delhi, India
}

Corresponding Author: Jyoti Bagla, Department of Pediatrics, ESI Hospital and PGIMSR, Basaidarapur, New Delhi, India, Phone: +91 9711719117, e-mail: jyotibagla.dr@gmail.com

How to cite this article: Kumari S, Bagla J, et al. Pediatric Obstructive Sleep Apnea: A Review of Approach to Management. Indian Sleep Med 2019;14(2):32-37.

Source of support: Nil

Conflict of interest: None

cardiopulmonary dysfunction. Polysomnography (PSG) is the definitive investigation that identifies the obstructive events and quantifies the severity of OSA.

\section{Questionnaire-based Assessment}

SRBD scale is a questionnaire-based scoring system containing 22 symptom-based items. Scores more than 0.33 (score ranges from 0 to 1 ) is classified as positive which is suggestive of high risk for sleep apnea. ${ }^{13}$ When compared to PSG, the accuracy of the SRBD score has been demonstrated to be acceptable for diagnosis of suspected OSA. ${ }^{14}$

\section{Polysomnography}

Polysomnography, also known as a sleep study, is an electrophysiological study with concurrent multiparameter recordings that intent to record the quality of sleep, arousal episodes, apneahypopnea episodes, and oxygenation. The American Academy of Sleep Medicine (AASM) scoring manual recommends composite use of electroencephalogram, electromyogram, electrooculogram, $\mathrm{ECG}$, oronasal airflow, end-tidal $\mathrm{CO}_{2}$, and oxygen saturation $\left(\mathrm{SpO}_{2}\right)$ to study sleep disorders. Apnea-hypopnea index (AHI), respiratory disturbance index (RDI), and degree of hypoventilation are derived from the parameters recorded during a sleep study. It is important to understand the difference between children and adults during a sleep study. Children require a friendly atmosphere and approach, need smaller and specialized equipment, and due to developmental 
Table 1: Evaluation of sleep apnea and therapeutic options in children

Evaluation of sleep apnea in children

- Focused sleep history

- Snoring

- Labored breathing or observed pauses in breathing during sleep

- Daytime attention, learning difficulties, and behavior problems

- Daytime sleepiness

- Physical examination including detailed examination of the oropharynx

- Growth assessment-obesity and poor growth

- Systemic or pulmonary hypertension

- Craniofacial anomalies

- Abnormal maxillomandibular development

- Direct laryngoscopy — tonsil and adenoid size estimation

- Investigation

- PSG

- Upper airway magnetic resolution imaging (MRI)/computed tomography (CT) scan

- Soft tissue neck X-ray (lateral/AP)

- Flexible nasopharyngoscopy

Therapeutic options for sleep apnea in children

- Lifestyle modification

- Medical therapy-noninvasive PAP

- Pharmacologic management-Montelukast and intranasal corticosteroids

- Surgical management-adenotonsillectomy, mandibular distraction, palatal expansion and distraction osteogenesis maxillary expansion

- Adjunct therapies

and physiological differences from adults, have age-adjusted rules for the scoring and interpretation of polysomnograms. ${ }^{12}$

\section{Assessment of Severity of Sleep Apnea}

Clinician takes help of polysomnographic study result to classify the severity of OSA as it determines both treatment options and outcome. $\mathrm{AHI}$ is a measurement derived from the PSG study which denotes the number of apneas plus hypopneas per hour of sleep.

Classification of sleep apnea severity in children based on the $\mathrm{AHI}$

Severity: AHI

Normal: $\leq 1$

Mild: $>1$ to $\leq 5$

Moderate: $>5$ to $\leq 10$

Severe: $>10$

\section{TREATMENT}

The therapeutic decision for a case of sleep apnea is taken after considering the clinical severity and the sleep study findings. Table 1 summarizes the therapeutic options in sleep-disordered breathing. An algorithmic approach suggested on the basis of PSG finding can be adopted for managing an OSA. Mild OSA can be managed with watchful waiting with lifestyle modification antiinflammatory medications and close follow-up. Moderate to severe sleep apnea needs surgery or positive airway therapy as given in detail subsequently (Flowchart 1).

\section{Conservative Management}

Mild OSA with minimal impairment in quality of life can be managed with watchful waiting with lifestyle modification and correction of obesity, if any.

\section{Diet and Lifestyle Modification}

Obesity is one of the risk factors that predisposes OSA. ${ }^{15}$ Correction of obesity is an important adjunctive measure for OSA management and also in preventing surgical treatment failure. Limited studies in pediatric population show weight loss to be effective in treating obese children with sleep-disordered breathing. ${ }^{16}$ Moreover, no obvious benefit is expected in nonobese children. Lifestyle modification including improvement in sleep hygiene, behavioral modification contributes to improving the quality of life along with the primary treatment options.

\section{Watchful Waiting}

Watchful waiting with care for coexisting morbidities can be an acceptable modality as compared to surgical intervention for mild to moderate severity of OSA diagnosed by the PSG study. Watchful waiting means a regular follow-up with an objective evaluation of disease severity. Although surgical intervention improves PSG findings, an acceptable percentage of children improve with watchful waiting alone as shown in the Childhood Adenotonsillectomy Trial (CHAT) by Marcus et al. ${ }^{17}$ Evidence from a recent meta-analysis shows normalization of abnormal PSG findings by 7 months with a watchful follow-up only in non-syndromic healthy children in the age group of 5-9 years diagnosed with mild to moderate OSAS by the PSG study. ${ }^{18}$

Surgical intervention definitely has greater improvement in symptomatology, particularly in behavioral abnormalities, sleep apnea symptoms, growth, and quality of life, so treatment modality should be tailored according to severity. Children with severe OSA should not be advised for watchful waiting. Adjunctive care must be given during this period to take care of comorbidities such as obesity, rhinitis, asthma, and poor sleep hygiene.

\section{Pharmacologic Treatment}

\section{Rationale}

Obstruction of upper airway secondary to nasal septum deviation and nasal turbinate hypertrophy by mucosal inflammation in allergic rhinitis increases the risk for habitual snoring and mildmoderate OSAS. Pharmacological intervention reduces mucosal inflammation, which in turn decrease mucosal edema and upper airway resistance.

Intranasal corticosteroid and leukotriene inhibitors are the medications used to reduce symptom burden in mild-to-moderate OSA. These are used as a temporary intervention while the watchful waiting period or as treatment of coexisting morbidity after adenotonsillectomy. Intranasal corticosteroids may significantly improve nasal obstruction symptoms in children with moderate to severe adenoidal hypertrophy, and this improvement may be associated with a reduction of adenoid size. ${ }^{19}$ Chan et al. studied the role of intranasal mometasone furoate in mild OSA in the 6-18 year age group and found it to reduce the severity by 4 months of intervention. ${ }^{20} \mathrm{~A}$ similar effect is also shown with intranasal budesonide as a nonsurgical alternative in mild pediatric OSA with its positive effects being sustained till 2 months after discontinuation of the drug. ${ }^{21}$ 
Flowchart 1: Algorithmic approach to management of pediatric OSA

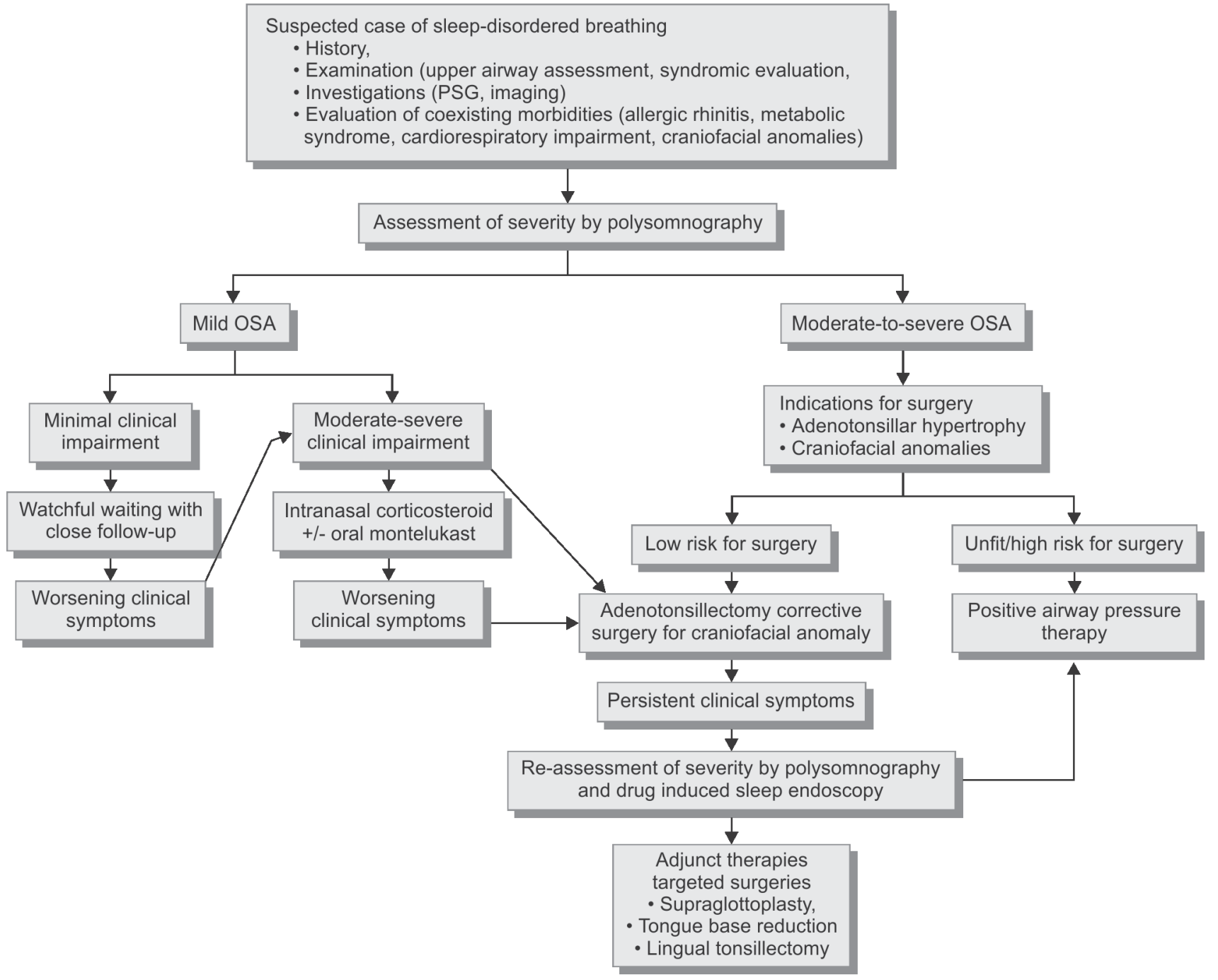

Leukotriene inhibitors are the second group of anti-inflammatory drugs thathave been studied in mild OSA and post-adenotonsillectomy patient with mild symptoms. Leukotriene receptors have been found to be upregulated in tonsillar hypertrophy, ${ }^{22,23}$ which can be targeted with these medications, thereby the decreasing obstructive effect in the upper airway. When used for nonsevere OSA in the age group of 2-10 years over 16 weeks duration, $\mathrm{AHI}$ was significantly decreased in the Montelukast group as compared to placebo. ${ }^{24}$

Preliminary evidences favor the use of the combination of intranasal corticosteroid and oral Montelukast to surgical intervention as initial treatment of mild OSA in younger, nonobese children, and residual OSA in the post-adenotonsillectomy patient. ${ }^{25,26}$

\section{Positive Airway Pressure}

\section{Rationale}

Positive airway pressure (PAP) therapy intends to maintain the patency of upper airway in obstructive pathology by application of continuous or cyclical noninvasive mechanical pressure. The use of airway pressure above the critical closing pharyngeal pressure helps in maintaining patency in collapsible pharyngeal musculature in obese children. ${ }^{27}$
There are two types of PAP modalities namely: continuous positive airway pressure (CPAP) and bilevel positive airway pressure (BPAP). CPAP utilizes the application of continuous positive pressure throughout the respiration, while BiPAP delivers differential predetermined pressures during inspiration and expiration. Auto-PAP is a modified CPAP system which self-titrates the pressure needed according to the pressure changes sensed during respiration in sleep to keep the airway patent. Required pressure values for a CPAP system are determined by a diagnostic PSG test. It remains an ideal method to decide pressure requirement; however, Auto-PAP can be used to adjust the pressure needs automatically while manual results are awaited. ${ }^{28}$

\section{Indications}

Positive airway pressure therapy is reserved for the children with moderate to severe OSA in the following:

- Obese children

- Syndromic children-a Down syndrome with hypotonia and craniofacial anomaly

- Postsurgical children with persistent symptoms

- Contraindication to surgery

- Strong patient preference for a nonsurgical approach

- As an adjunctive measure in the postoperative phase 


\section{Contraindication}

PAP therapy is difficult to adhere to young children, claustrophobic children. It is not considered safe to use in a patient with profuse oral secretion due to the risk of aspiration. Bilevel PAP is indicated in children with hypoventilation proven by the sleep study.

Published literature regarding PAP therapy mainly comprises of observational studies evaluating effectiveness and compliance. Continuous positive airway pressure was shown to be useful in $86 \%$ of cases in the age group of 6-19years. ${ }^{29}$ The use of PAP therapy has been shown to reduce nighttime $\mathrm{AHI}$ episodes from a pretreatment mean of 27 episodes/hour to 3 episodes/hour with improvement in daytime symptoms. ${ }^{30}$ The use of PAP therapy can be used as a respiratory support in high-risk adenotonsillectomy patients to avoid the risks of intubation and ventilator dependence. ${ }^{31}$

Compliance remains the sole challenge in maintaining PAP therapy in young children and children with developmental disorders or behavior disorders. Various attempts to improve tolerability and adherence of CPAP therapy have proven ineffective. Behavioral therapy, including desensitization, by positive reinforcement, graduated exposure, and counter-conditioning are some of the methods to improve compliance. ${ }^{32}$ Trials of bilevel positive airway pressure, auto-titrating positive airway pressure, and comfort features, such as pressure relief during exhalation, all demonstrate efficacy but adherence remains poor. High flow nasal cannula (HFNC) has been proven to be an effective and tolerable CPAP alternative for the treatment of pediatric OSA. Studies have demonstrated that HFNC reduces respiratory events, improves oxygenation, reduces heart rate, and does not disturb sleep quality in pediatric patients with moderate to severe OSA who have not tolerated CPAP. ${ }^{33,34}$

\section{Complication}

Minor complications can be encountered due to improper interface application and improper humidification. Conjunctivitis, skin damage at contact, skin hypo- or hyperpigmentation, mask discomfort, and claustrophobia are a few of the problems encountered. Pressure and gas flow-induced aerophagia may lead to bloating, gaseous abdomen distension, and excessive gas passing. ${ }^{35}$

\section{Surgical Therapy}

\section{Rationale}

Overgrowth of adenotonsillar tissue obstructs the upper airway and surgical removal improves obstructive symptoms. Surgical correction of other craniofacial anomalies in syndromic children helps in stabilizing the upper airway. Surgical intervention remains the first-line of treatment in moderate to severe OSA with adenotonsillar hypertrophy.

Following are the surgical procedures related to the management of sleep apnea:

- Adenotonsillectomy

- Expansion sphincter pharyngoplasty

- Orthodontic surgery

- Lingual tonsillectomy

- Supraglottoplasty

- Tongue base suspension or reduction

\section{Adenotonsillectomy}

American Academy of Otolaryngology-Head and Neck Surgery (AAO-HNS) recommends adenotonsillectomy as the first-line treatment for OSA in otherwise healthy children over 2 years of age with adenotonsillar hypertrophy. ${ }^{36}$

Indications

- Children with adenotonsillar hypertrophy and severe OSA

- Children with moderate OSA

- Children with moderate OSA if watchful waiting fails to improve symptoms

- Syndromic children with OSA as in Down syndrome and craniofacial anomalies

The presence of a submucous cleft palate can be considered as a relative contraindication to adenoidectomy.

Evidences: a prospective longitudinal study by Huang et al. has shown a significant improvement in $\mathrm{AHI}$ at 6 months with a mean decrease in $\mathrm{AHI}$ episodes by 10 episodes/hour. However, this improvement was decreased by 36 months postoperative in $68 \%$ of the patients. ${ }^{37}$ Adenotonsillectomy results in marked improvements in the quality of life, PSG study findings, and behavioral issues which are sustained in the long-term also. ${ }^{38}$

The role of AT is questionable in obese children as the surgery improves the symptoms significantly by $\mathrm{AHI}$ reduction of 18.3 events per hour but complete resolution of symptom is observed in $12 \%$ of cases as per one of the metanalyses. ${ }^{39}$

Adenotonsillectomy is a minor surgical procedure done under general anesthesia with the use of a curette, microdebrider, or suction electrocautery. A bit of adenoid tissue is left inferiorly to take care of velopharyngeal insufficiency. Risk of adenoid regrowth remains for the age group of less than 2 years.

Complications of surgery include bleeding, infection, anesthesia-related complications, velopharyngeal incompetence, and subglottic stenosis.

Follow-up after adenotonsillectomy: The follow-up after 6-8 weeks postsurgery should be ensured to assess the symptoms or signs of OSA. ${ }^{40}$ Repeated PSG should be done to reevaluate the severity of their sleep-related breathing abnormalities and need for further therapeutic interventions including positive airway pressure therapy, in children with complications of OSA, and OSA with syndromes.

\section{Expansion Sphincter Pharyngoplasty}

This is the repair of pharyngeal wall musculature in the case of lateral pharyngeal wall collapse detected by sleep endoscopy. When compared to adenotonsillectomy, modified expansion sphincter pharyngoplasty had a higher percentage of cure rate and better resolution of $\mathrm{AHI}$ episodes in the presence of lateral pharyngeal weakness. $^{41}$

\section{Orthodontics}

Rationale: OSA affected children with insignificant tonsillar hypertrophy and dental malocclusion or narrow palate benefit from the widening of the maxillary bone. This widening achieved through distraction osteogenesis by rapid maxillary expansion results in increased airway patency and resolution of nocturnal obstruction.

Rapid maxillary expansion (RME) is typically preferred before puberty when the maxilla fuses in the midline. RME has been shown to decrease the severity of OSA in nonobese children with maxillary contraction, the effect being sustained at 3 years postintervention. ${ }^{42,43}$

\section{Miscellaneous Therapies}

A few of the following interventions can be beneficial as an adjunct to the primary surgical or medical intervention: 
- Environmental pollutants-Smoke, dust, and other irritants can aggravate the upper airway reactivity and worsen obstruction. Avoidance of irritants, normal saline irrigation, and education should complement the specific anti-inflammatory therapy.

- Supplemental oxygen-Normalization of hypoxemic episodes by oxygen therapy should be targeted till definite therapy. This is necessary for symptomatic unfit candidates.

- Myofascial reeducation-This is a type of orofacial breathing exercises that improve the tongue and orofacial muscle strength. This approach is adapted from its success among the adult patients who have a failure of PAP therapy or surgery. The beneficial effect has been seen in a few studies, ${ }^{44-46}$ but more prospective studies with adequate subjects are needed to adopt it as a primary or complementary noninvasive intervention.

- Nasal expiratory positive airway pressure (NEPAP) device is a wearable device which has an one-way valve that produces an expiratory resistance. Variable results observed in a pilot study of 14 subjects keep it as an experimental device only. ${ }^{47}$

\section{Management of Persistent OSA}

Despite being the primary intervention in pediatric OSA, adenotonsillectomy fails to achieve success in approximately $11-24 \%$ of the patient treated. Follow-up studies after surgery identify the persistent OSA and such patients should be subjected to further investigations like drug-induced sleep endoscopy (DISE) to detect a reason for failure. Lingual tonsillar hypertrophy and occult laryngomalacia are among the few of conditions causing persistent OSAS after surgery. ${ }^{48}$ Tongue base suspension or reduction, supraglottoplasty, and lingual tonsillectomy are the corrective surgeries according to the condition diagnosed.

\section{Conclusion}

Pediatric sleep apnea, an often ignored entity, needs a high index of suspicion and investigation to diagnose the severity and existing comorbidities. There is a need for sensitization of pediatricians and respiratory physicians so as to help them to promptly recognize and intervene. Adenotonsillectomy remains the primary surgery for severe cases whereas positive airway pressure and pharmacological therapy are a good alternative option when surgery is not possible. Close follow with multidisciplinary intervention is needed to restore functional activities in sleep-disordered breathing.

\section{Acknowledgment}

The authors would like to express their gratitude to Dr Dipti Gothi, Professor and Head, Department of Pulmonary Medicine, ESI Hospital PGIMSR, Basaidarapur, Delhi, for providing valuable inputs during the compilation of the review.

\section{References}

1. American Academy of Sleep Medicine. International Classification of Sleep Disorders, 3rd ed., Darien, IL: American Academy of Sleep Medicine; 2014.

2. American Thoracic Society. Standards and indications for cardiopulmonary sleep studies in children. Am J Respir Crit Care Med 1996 Feb;153(2):866-878. DOI: 10.1164/ajrccm.153.2.8564147.

3. Bixler EO, Vgontzas AN, et al. Sleep disordered breathing in children in a general population sample: prevalence and risk factors. Sleep 2009 Jun;32(6):731-736. DOI: 10.1093/sleep/32.6.731.
4. Li AM, So HK, et al. Epidemiology of obstructive sleep apnoea syndrome in Chinese children: a two-phase community study. Thorax 2010;65(11):991-997. DOI: 10.1136/thx.2010.134858.

5. O'Brien LM, Holbrook CR, et al. Sleep and neurobehavioral characteristics of 5- to 7-year-old children with parentally reported symptoms of attention-deficit/hyperactivity disorder. Pediatrics 2003 Mar;111(3):554-563. DOI: 10.1542/peds.111.3.554.

6. Huang YS, Guilleminault C. Pediatric Obstructive Sleep Apnea: Where Do We Stand? Adv Otorhinolaryngol 2017;80:136-144. DOI: 10.1159/000470885.

7. Mallampati SR, Gatt SP, et al. A clinical sign to predict difficult tracheal intubation: a prospective study. Can Anaesth Soc J 1985 Jul;32(4):429-434. DOI: 10.1007/BF03011357.

8. Friedman M, Salapatas AM, et al. Updated Friedman Staging System for Obstructive Sleep Apnea. Adv Otorhinolaryngol 2017;80:41-48. DOI: $10.1159 / 000470859$.

9. Kumar HV, Schroeder JW, et al. Mallampati score and pediatric obstructive sleep apnea. J Clin Sleep Med 2014 Sep 15;10(9):985-990. DOI: $10.5664 / \mathrm{jcsm} .4032$.

10. Trivedi M, ElMallah $M$, et al. Pediatric Obstructive Sleep Apnea and Asthma: Clinical Implications. Pediatr Ann 2017 Sep 1;46(9): e332-e335. DOI: 10.3928/19382359-20170815-03.

11. Park DY, Choi JH, et al. Correlations between pediatric obstructive sleep apnea and longitudinal growth. Int J Pediatr Otorhinolaryngol 2018 Mar;106:41-45. DOI: 10.1016/j.ijporl.2018.01.001.

12. Beck SE, Marcus CL. Pediatric polysomnography. Sleep Med Clin 2009 Sep;4(3):393-406. DOI: 10.1016/j.jsmc.2009.04.007.

13. Chervin RD, Hedger $K$, et al. Pediatric sleep questionnaire (PSQ): validity and reliability of scales for sleep-disordered breathing, snoring, sleepiness, and behavioral problems. Sleep Med 2000 Feb 1;1(1):21-32. DOI: 10.1016/S1389-9457(99)00009-X.

14. Michelet $D$, Julien-Marsollier F, et al. Accuracy of the sleep-related breathing disorder scale to diagnose obstructive sleep apnea in children: a meta-analysis. Sleep Med 2019 Feb;54:78-85. DOI: 10.1016/j.sleep.2018.09.027.

15. Kohler $\mathrm{M}$, Lushington $\mathrm{K}$, et al. Obesity and risk of sleep related upper airway obstruction in Caucasian children. J Clin Sleep Med 2008 Apr 15;4(2):129-136. DOI: 10.1159/000470859.

16. Van Hoorenbeeck K, Franckx H, et al. Weight loss and sleepdisorderedbreathing in childhood obesity: effects on inflammation and uric acid. Obesity (Silver Spring) 2012 Jan;20(1):172-177. DOI: 10.1038/oby.2011.282.

17. Marcus $\mathrm{CL}$, Moore RH, et al. Childhood Adenotonsillectomy Trial (CHAT). A randomized trial of adenotonsillectomy for childhood sleep apnea. N Engl J Med 2013 Jun 20;368(25):2366-2376. DOI: 10.1056/ NEJMoa1215881.

18. Venekamp RP, Hearne BJ, et al. Tonsillectomy or adenotonsillectomy versus non-surgical management for obstructive sleep-disordered breathing in children. Cochrane Database Syst Rev 2015 Oct; 14(10):CD011165. DOI: 10.1002/14651858.CD011165.pub2.

19. Zhang L, Mendoza-Sassi RA, et al. Intranasal corticosteroids for nasal airway obstruction in children with moderate to severe adenoidal hypertrophy. Cochrane Database Syst Rev 2008 Jul;16(3):CD006286. DOI: 10.1002/14651858.CD006286.pub2.

20. Chan CC, Au CT, et al. Intranasal corticosteroids for mild childhood obstructive sleep apnea-a randomized, placebo-controlled study. Sleep Med 2015 Mar;16(3):358-363. DOI: 10.1016/j.sleep.2014.10.015.

21. Kheirandish-Gozal L, Gozal D. Intranasal budesonide treatment for children with mild obstructive sleep apnea syndrome. Pediatrics 2008 Jul;122(1):e149-e155. DOI: 10.1542/peds.2007-3398.

22. Tsaoussoglou M, Lianou $L$, et al. Cysteinyl leukotriene receptors in tonsillar B- and T-lymphocytes from children with obstructive sleep apnea. Sleep Med 2012 Aug;13(7):879-885. DOI: 10.1016/ j.sleep.2012.03.010.

23. Tsaoussoglou M, Hatzinikolaou S, et al. Expression of leukotriene biosynthetic enzymes in tonsillar tissue of children with obstructive sleep apnea: a prospective nonrandomized study. JAMA Otolaryngol 
Head Neck Surg 2014 Oct;140(10):944-950. DOI: 10.1001/jamaoto. 2014.1936.

24. Kheirandish-Gozal L, Bandla HP, et al. Montelukast for Children with Obstructive Sleep Apnea: Results of a Double-Blind, Randomized, Placebo-Controlled Trial. Ann Am Thorac Soc 2016 Oct;13(10): 1736-1741. DOI: 10.1513/AnnalsATS.201606-432OC.

25. Kheirandish-Gozal L, Bhattacharjee R, et al. Antiinflammatory therapy outcomes for mild OSA in children. Chest 2014 Jul;146(1):88-95. DOI: 10.1378/chest.13-2288.

26. Kheirandish L, Goldbart AD, et al. Intranasal steroids and oral leukotriene modifier therapy in residual sleep-disordered breathing after tonsillectomy and adenoidectomy in children. Pediatrics 2006 Jan;117(1):e61-e66. DOI: 10.1542/peds.2005-0795.

27. Huang J, Pinto SJ, et al. Upper airway collapsibility and genioglossus activity in adolescents during sleep. Sleep 2012 Oct 1;35(10): 1345-1352. DOI: 10.5665/sleep.2110.

28. Mihai $R$, Vandeleur $M$, et al. Autotitrating CPAP as a Tool for CPAP Initiation for Children. J Clin Sleep Med 2017 May 15;13(5):713-719. DOI: $10.5664 / \mathrm{jcsm} .6590$.

29. Marcus $\mathrm{CL}$, Ward SL, et al. Use of nasal continuous positive airway pressure as treatment of childhood obstructive sleep apnea. J Pediatr 1995 Jul;127(1):88-94. DOI: 10.1016/S0022-3476(95)70262-8.

30. Marcus CL, Rosen G, et al. Adherence to and effectiveness of positive airway pressure therapy in children with obstructive sleep apnea. Pediatrics 2006 Mar;117(3):e442-e451. DOI: 10.1542/peds.2005-1634.

31. Friedman $\mathrm{O}$, Chidekel $\mathrm{A}$, et al. Postoperative bilevel positive airway pressure ventilation after tonsillectomy and adenoidectomy in children-a preliminary report. Int J Pediatr Otorhinolaryngol 1999 Dec 15;51(3):177-180. DOI: 10.1016/S0165-5876(99)00260-8.

32. Chervin RD, Theut $S$, et al. Compliance with nasal CPAP can be improved by simple interventions. Sleep 1997 Apr;20(4):284-289. DOI: $10.1093 /$ sleep/20.4.284.

33. Hawkins S, Huston S, et al. High-Flow, Heated, Humidified Air Via Nasal Cannula Treats CPAP-Intolerant Children With Obstructive Sleep Apnea. J Clin Sleep Med 2017 Aug 15;13(8):981-989. DOI: 10.5664/ jcsm.6700.

34. Joseph L, Goldberg S, et al. High-Flow Nasal Cannula Therapy for Obstructive Sleep Apnea in Children. J Clin Sleep Med 2015 Sep 15;11(9):1007-1010. DOI: 10.5664/jcsm.5014.

35. Guilleminault C, Nino-Murcia G, et al. Alternative treatment to tracheostomy in obstructive sleep apnea syndrome: nasal continuous positive airway pressure in young children. Pediatrics 1986 Nov;78(5):797-802.
36. Mitchell RB, Archer SM, et al. Clinical Practice Guideline: Tonsillectomy in Children (Update). Otolaryngol Head Neck Surg 2019 Feb; 160(Suppl 1):S1-S42.

37. Huang YS, Guilleminault C, et al. Treatment outcomes of adenotonsillectomy for children with obstructive sleep apnea: a prospective longitudinal study. Sleep 2014 Jan 1;37(1):71-76. DOI: 10.5665/sleep. 3310 .

38. Baldassari CM, Mitchell RB, et al. Pediatric obstructive sleep apnea and quality of life: a meta-analysis. Otolaryngol Head Neck Surg 2008 Mar;138(3):265-273. DOI: 10.1016/j.otohns.2007.11.003.

39. Costa DJ, Mitchell R. Adenotonsillectomy for obstructive sleep apnea in obese children: a meta-analysis. Otolaryngol Head Neck Surg 2009;140:455-460. DOI: 10.1016/j.otohns.2008.12.038.

40. Marcus $\mathrm{CL}$, Brooks LJ, et al. American Academy of Pediatrics. Diagnosis and management of childhood obstructive sleep apnea syndrome. Pediatrics 2012 Sep;130(3):576-584. DOI: 10.1542/peds.2012-1671.

41. Ulualp SO. Modified expansion sphincter pharyngoplasty for treatment of children with obstructive sleep apnea. JAMA Otolaryngol Head Neck Surg 2014;140(9):817-822. DOI: 10.1001/jamaoto.2014.1329.

42. Pirelli P, Saponara M, et al. Rapid maxillary expansion in children with obstructive sleep apnea syndrome. Sleep 2004 Jun 15;27(4):761-766. DOI: 10.1093/sleep/27.4.761.

43. Villa MP, Malagola C, et al. Rapid maxillary expansion in children with obstructive sleep apnea syndrome: 12-month follow-up. Sleep Med 2007 Mar;8(2):128-134. DOI: 10.1016/j.sleep.2006.06.009.

44. Villa MP, Evangelisti M, et al. Can myofunctional therapy increase tongue tone and reduce symptoms in children with sleep-disordered breathing? Sleep Breath 2017 Dec;21(4):1025-1032. DOI: 10.1007/ s11325-017-1489-2.

45. Guilleminault C, Huang YS, et al. Critical role of myofascial reeducation in pediatric sleep-disordered breathing. Sleep Med 2013 Jun;14(6):518-525. DOI: 10.1016/j.sleep.2013.01.013.

46. Villa MP, Brasili L, et al. Oropharyngeal exercises to reduce symptoms of OSA after AT. Sleep Breath 2015 Mar;19(1):281-289. DOI: 10.1007/ s11325-014-1011-z.

47. Kureshi SA, Gallagher PR, et al. Pilot study of nasal expiratory positive airway pressure devices for the treatment of childhood obstructive sleep apnea syndrome. J Clin Sleep Med 2014 Jun 15;10(6):663-669. DOI: $10.5664 /$ jcsm.3796.

48. Chan DK, Jan TA, et al. Effect of obesity and medical comorbidities on outcomes after adjunct surgery for obstructive sleep apnea in cases of adenotonsillectomy failure. Arch Otolaryngol Head Neck Surg 2012 Oct;138(10):891-896. DOI: 10.1001/2013.jamaoto.197. 